\title{
Influential Statements and Gaze for Persuasion Modeling
}

\author{
Hana Vrzakova \\ School of Computing \\ University of Eastern Finland \\ Joensuu, Finland \\ hanav@uef.fi \\ Roman Bednarik \\ School of Computing \\ University of Eastern Finland \\ Joensuu, Finland \\ roman.bednarik@uef.fi
}

Yukiko Nakano

Seikei University

Fumio Nihei

Seikei University

Kichijoji-Kitamachi, Musashino Kichijoji-Kitamachi, Musashino

Tokyo, Japan

Tokyo, Japan

y.nakano@st.seikei.ac.jp

dm146211@cc.seikei.ac.jp
Permission to make digital or hard copies of part or all of this work for persona or classroom use is granted without fee provided that copies are not made or and the full citation on the first page. Copyrights for third party compotice and the full citation on the first page. Copyrights for third-party components of this work must be honored. For all other Copyight is held by the ow 14 ,

978-1-4503-2542-4/14/10. http://dx.doi.org/10.1145/2639189.2670192

\begin{abstract}
Influential statements during conversations change the flow of the discussion and open new directions in the conversation. The content of the statement does not make the statement influential alone, it is strengthened by behavioral patterns, such as voice pitch, facial gestures, gaze and body postures. In this work we focus on the relationship between influential statements and gaze, as a potential cue in the automatic detection of conversation skills and in replicating natural interaction behavior for companionship and persuasive technologies. Within a multimodal data corpus of group conversations, we present an approach to analysis of the rich social signals and explore the potentials for correlation between the influential statements and gaze. The statements in the conversations were semi-automatically annotated and scored according to the level of influence, which provided us with boundaries for the gaze analysis. We present the first results of this approach.
\end{abstract}

\section{Author Keywords}

Influential statement, Conversation, Gaze, CSCW,

Persuasive technologies

\section{ACM Classification Keywords}

H.5.2 [User Interfaces]: Miscellaneous. 


\section{Multimodal group}

discussions corpus

Participants: 40 university students, 11 female, 29 male, Mean age $=23$ years $(S D=3)$

Recordings: 10 groups, 4 participants per group, 40 hours of recordings

Apparatus: Motion capture system for hand gestures; acceleration sensors were used for head gestures; Kinect system to detect overall body posture; personal microphone and Tobii eye-tracking glasses. Figure 1 shows the final setup from the overall scene camera.

Tasks in the session: Find a shared agreement during a) booth-planning, b) planning a travel for your foreign friend, and c) selecting a celebrity for the school festival. Each tasks has to be accomplished in 20 minutes.

Motivation: Fundamental conversational skills, as proposing an idea, discussing the statements and making the decision, are equally distributed in each phase of the discussion and promote occurrence of influential statements.

\section{Introduction}

One of the aspects of group conversation management is the flow of influential statements and persuasion abilities. Influence on a conversation is not necessarily connected only to the participant's knowledge, but it is also the sense for timing, and the social signals such as body posture, voice characteristics, eye contact, to name a few contributing factors that comprise the conversation management.

Contrary to face-to-face conversations, in video conferencing, distant education, remote team-work and other computer-mediated collaboration environments, the aforementioned social signals are muted by technology. The remote and virtual interaction cuts away the perfect face-to-face sensory experience of the participants, and limits the originally-rich conversation to the video stream on a screen with limited view, sound, and depth of perception. The gap in the user experience is even more eminent when the interaction becomes multi-party; the participants in the conversation have to exercise even more efforts to understand each other and to influence others through their statements and behavior.

Agent-based technologies present possibilities in bridging the lack in the sensory user experience. For instance, Andrist et al. modelled the gaze aversion to reproduce intentional human-like behaviors for a conversational robot [1]. Similar technologies could be used to detect and model various aspects of conversation management such as the influential statements, and could exhibit persuasive behaviors. For example, an intelligent system could monitor a conversation and upon sensing persuasive behaviors could change the estimation of the participant's conversational skills or could improve the remote interaction by strengthening the relevant cues.
Our approach is however cautious. Before we enhance computer mediated conversations and persuasive technology with the ability to detect and produce influential statements, we need to gain better understanding of what makes statements influential and which social signals are the most important to support the natural-like influence. Influential statement is defined in this work as a utterance of a person which provoked a cascade of others' ideas and follow-up statements. In our prior research authors analyzed such cascades and related them to the voice characteristics [6]. In this work we focus on the relationship influential statements and between gaze.

\section{Background}

In previous studies of group discussions, multimodal signals, such as speech, gaze and gestures, have been employed to estimate the characteristics of individual participants. Sanchez-Cortes et al. [7] proposed a model for predicting emergent leadership, and found that dominance and leadership was highly correlated. Audio-visual nonverbal features were also used for predicting personality impressions [2].

However, all these studies have been focused on characteristics of the individual as they omitted the effects of interaction in a group. In the group interaction the personal characteristics can change due to the dynamics of interaction, and thus it is necessary to detect influential statements that affect the group dynamics. For this purpose, our work focuses on eye gaze information as potential predictor of influential statements.

Simultaneous gazing or the mutual gaze by the speakers is important when agreeing on the speaker change [4]. Verteegal et al. also examined gaze direction during 


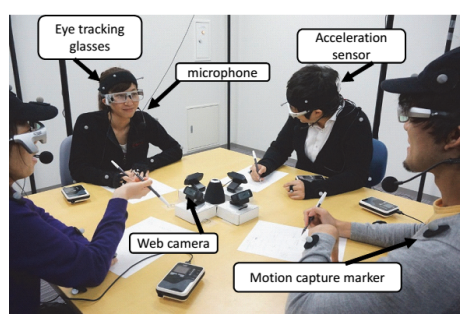

Figure 1: Recording systems employed during experiments.

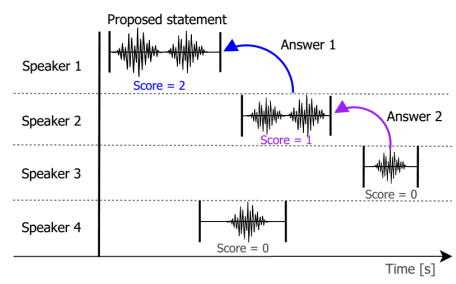

Figure 2: Score influential statements. Each statements is scored according to the number of answers. More answers the idea received the higher score is. multiparty human-human interaction [8]. Bednarik et al employed gaze patterns for automatic classification of conversational engagement in remote group discussions [3] .

Since gaze plays an important role in social encounters [4], in this work we propose an extension to the current state of the art by observing characteristics of gaze during influential statements, and discuss whether the gaze information can be a useful predictor of such statements.

\section{Detecting influential statements: Dataset and} methodological challenges

In the collected multimodal corpus of multi-party conversations [6], participants formed groups of four and discussed the given in-basket question using detailed materials related to the task, for example Where is the best place for a booth for upcoming school festival? with a provided map of the school campus and details about the school festival. Within 20 minutes the participants had to come up with a solution through shared agreement. The discussion tasks were designed to cover analytical thinking, negotiation and decision making of the participants.

All statements were than annotated using the score of influence, where the score expresses a number of received answers and follow-up utterances. The statement was categorized as influential when it received a score equal or higher than two. Figure 2 illustrates a system of the score computation according to statement dependencies.

\section{Gaze patterns in influential statements}

Figure 3 displays an aggregated visualization of gaze for speakers during influential statements and non-influential statements. These preliminary results indicate that there are differences in gaze patterns contingent on the level of influence a statement had in the conversation. When the speaker said a persuasive comment, his gaze was coherent, and typically in one direction. In comparison, during non-influential statements, such as replies, gaze was distributed more across the peers.

Traditional eye-tracking measures were not applied, because the low sampling rate of Tobii glassed did not allow to apply a reliable fixation identification for traditional eye movement analysis. We, however, are developing alternative methods to analyze the rich data.

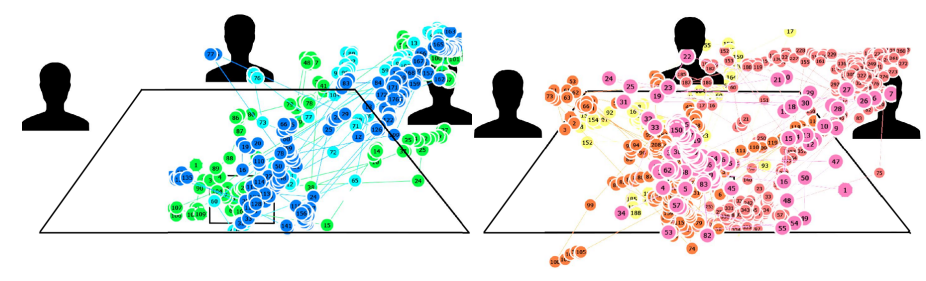

(a) Influential

(b) Casual

Figure 3: Comparison of gaze of a speaker for influential statements and non-influential statements. The figures are aggregated over three and four statements, respectively, and do not represent a constant time window.

\section{Conclusions}

Although preliminary, the results presented here show interesting differences in gaze for two levels of influential statements. The gaze paths during the influential statements revealed stronger attention orientation toward a single listener, while during the non-influential statements speaker's gaze was distributed to all listeners. This is an interesting observation since in a similar study of dominance and gaze, the dominant speaker exhibited a distributed gaze equally to all listeners during leadership 
statements [5]. This contradicting observation motivates further research to reveal the deeper relationships between influential statements and gaze behavior.

The type of analysis presented in this paper lays foundations for future research into the modeling of persuasive behavior in group conversations. Our aim is to extend the breadth of the analysis as well as to perform a multimodal data analysis. It will be possible to compare the relative contribution of each of the captured modalities to the detectability of influential statements. Moreover our aim is to compile the behaviors typical for persuasion into actionable models and let virtual or physical companionship agents to display these behaviors.

\section{References}

[1] Andrist, S., Tan, X. Z., Gleicher, M., and Mutlu, B. Conversational gaze aversion for humanlike robots. In Proc.of the 2014 ACM/IEEE Int. Conf. on Human-robot Interaction, HRI '14, ACM (New York, NY, USA, 2014), 25-32.

[2] Aran, O., and Gatica-Perez, D. One of a kind inferring personality impressions in meetings. In Proc. of the 15th ACM on Int. conf. on multimodal interaction, ACM (2013), 11-18.

[3] Bednarik, R., Eivazi, S., and Hradis, M. Gaze and conversational engagement in multiparty video conversation: an annotation scheme and classification of high and low levels of engagement. In Proc. of the 4th workshop on eye gaze in intelligent human machine interaction, ACM (2012), 10.

[4] Kendon, A. Some functions of gaze-direction in social interaction. Acta psychologica 26 (1967), 22-63.

[5] Nakano, Y., and Fukuhara, Y. Estimating conversational dominance in multiparty interaction. In Proc. of the 14th ACM Int. conf. on Multimodal interaction, ACM (2012), 77-84.

[6] Nihei, F., Nakano, Y., Hayashi, Y., Huang, H.-H., and Okada, S. Predicting influential statements in group discussions using speech and head motion information. In Proc. of the 16th ACM on International Conf. on Multimodal Interaction, ICMI '14, ACM (New York, NY, USA, 2014).

[7] Sanchez-Cortes, D., Aran, O., Jayagopi, D. B., Mast, M. S., and Gatica-Perez, D. Emergent leaders through looking and speaking: from audio-visual data to multimodal recognition. Journal on Multimodal User Interfaces 7, 1-2 (2013), 39-53.

[8] Vertegaal, R., Slagter, R., Van der Veer, G., and Nijholt, A. Eye gaze patterns in conversations: there is more to conversational agents than meets the eyes. In Proc. of the SIGCHI conf. on Human factors in computing systems, ACM (2001), 301-308. 